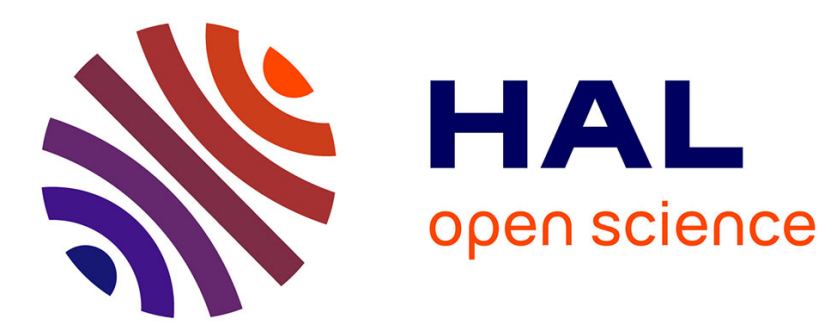

\title{
Collective Friction of an Array of Particles: A Crucial Test for Numerical Algorithms
}

\author{
Farhang Radjai, Jochen Schäfer, Sabine Dipple, Dietrich Wolf
}

\section{To cite this version:}

Farhang Radjai, Jochen Schäfer, Sabine Dipple, Dietrich Wolf. Collective Friction of an Array of Particles: A Crucial Test for Numerical Algorithms. Journal de Physique I, 1997, 7 (9), pp.1053-1070. 10.1051/jp1:1997109 . jpa-00247382

\section{HAL Id: jpa-00247382 https://hal.science/jpa-00247382}

Submitted on 1 Jan 1997

HAL is a multi-disciplinary open access archive for the deposit and dissemination of scientific research documents, whether they are published or not. The documents may come from teaching and research institutions in France or abroad, or from public or private research centers.
L'archive ouverte pluridisciplinaire HAL, est destinée au dépôt et à la diffusion de documents scientifiques de niveau recherche, publiés ou non, émanant des établissements d'enseignement et de recherche français ou étrangers, des laboratoires publics ou privés. 


\title{
Collective Friction of an Array of Particles: A Crucial Test for Numerical Algorithms
}

\author{
Farhang Radjai $\left({ }^{*}\right)$, Jochen Schäfer, Sabine Dippel and Dietrich Wolf \\ HLRZ, Forschungszentrum, 52425 Jülich, Germany
}

(Received 22 July 1996, revised 9 April 1997, accepted 30 May 1997)

PACS.81.05.Rm - Porous materials; granular materials
PACS.46.10.+z - Mechanics of discrete systems

\begin{abstract}
Using different simulation techniques, we study the influence of the friction law governing interparticle and particle-plane contacts on the dynamics of a regular array of rigid parallel cylinders moving on a plane. We find that the evolution of the system depends strongly on the simulation method. But both in simulations with a Contact Dynamics algorithm, which prescribes the basic Coulomb's law of friction, and in simulations with molecular dynamics, which prescribes a regularized form of Coulomb's law, a steady state with very similar collective rotation modes is reached. As long as the static and dynamic friction coefficients are equal, the rotation modes are independent of the initial conditions. We show that in all cases, the global steady-state coefficient of friction between the array and the plane is a function of the driving force and the system size, and hence is not Coulombian.
\end{abstract}

\section{Introduction}

The physical basis of the frictional properties of dense granular materials, studied first by Coulomb over two centuries ago [1], is still an open question [2-4]. The frictional resistance inside a (dense) granular system or along a bounding solid surface is a collective process involving the disordered network of interparticle contacts. In other words, due to kinematic correlations the particles do not contribute independently to the overall friction.

These correlations appear not only as a result of the multi-contact nature of a dense granular system, but also from the possibility of particles to roll over one another. At such nonsluding (NS) contacts no dissipation takes place. Moreover, according to Coulomb's law of friction (see below), the friction force $T$ at a NS contact is only "partially mobilized", i.e. it takes an absolute value between zero and the product of the coefficient of friction $\mu$ and the normal contact force $N$. Only at sliding (S) contacts, the friction force is fully mobilized (i.e. $\left.T=-\mu \operatorname{sgn}\left(v^{t}\right) N\right)$, where $v^{t}$ is the relative tangential velocity of the surfaces. For these reasons, the global frictional resistance is not expected to be a simple function of the interparticle coefficient of friction. It depends on the distribution of S and NS contacts in the medium and on the highly heterogeneous distribution of contact forces, as observed in experiments and numerical simulations [5-7].

$\left({ }^{*}\right)$ Author for correspondence (e-mail: radjai@comphys.uni-duisburg.de) 
If all particles could roll over one another, a granular assembly would deform without resistance, in contrast to the fact that a finite force is needed to trigger or to maintain a granular flow. Hence, the resistance of a dense granular system results essentially from the frustration of particle rotations. This means that, due to kinematic constraints, contacts cannot all be simultaneously NS. Consider, for instance, three identical disks mutually in contact. If the particles in this simple configuration rotate, then at least one of the three contacts has to be sliding. The patterns of rotational frustration are generally much more complex and need a systematic study if a fundamental understanding of the mechanisms of friction in granular systems is to be gained.

In this paper we are interested in the dynamics of an array of identical disks (or cylinders) supported by a basal straight line (or plane), set to motion by applying a constant force on one of its ends (see Fig. 1). Some aspects of this model have been studied in [8]. There, it is numerically shown that with the basic Coulomb's law of friction, the system self-organizes itself to reach a steady state that is independent of initial conditions. This final state involves a well-defined organization of forces and particle angular velocities, giving rise to (up to three) mesoscopic scales along the array. In the steady state, one can define a global coefficient of friction which is independent of initial conditions but depends on the driving force. The same reference, by an analytical study of the correlations between the rotation modes and the global steady-state friction, shows that even for this regular multi-contact system, the mechanisms leading to an overall friction are far more complicated than what might be expected on a purely intuitive basis.

An original feature of the simulation method employed in [8], namely the Contact Dynamics (CD) method, is to prescribe the exact Coulomb's law of friction and the condition of perfectly rigid particles. However, the physical behavior of the system, briefly presented above, would have been partially different if another simulation method was used. In fact, in more common algorithms, such as Molecular Dynamics (MD), a "regularized" form of Coulomb's friction is implemented, and the particles are not considered as rigid. Thus, the important point is to examine to what extent such a regularization may affect the macroscopic behavior of the system. Another possible friction law is the Coulomb's law with a static coefficient of friction in addition to a different dynamic one. How would the dynamics of the system be influenced by such a law? This paper is concerned in the first place with these issues. We discuss in detail the ability of the numerical algorithms used to describe correctly the physics of the system. We study the time evolution, and more particularly the mechanisms of self-organization (essentially missing in [8]), involving a new effect due to friction with the driving block. Furthermore, the influence of mechanical parameters, such as the local coefficients of friction at particle-particle and particle-plane contacts, on the global coefficient of friction are systematically investigated. For the sake of self-containedness, some overlaps with [8] could not be avoided.

We will first introduce the model and its parameters. The friction laws and their prescription in numerical codes are discussed next. The most important results are grouped in three sections concerning the evolution, the steady-state collective modes and the steady-state friction. We will conclude with a discussion of the most salient physical features of the model and the relevance of the numerical algorithms. Some technical details have been placed in appendices.

\section{The Model}

Figure 1 shows the geometry of a linear array of identical disks on a straight line. The particles are numbered from 1 to $L$ opposite to the direction of motion. A horizontal driving force $N_{L}$ is applied to the particle $L$ by a "pushing block". At the other end of the array, a braking force $-N_{0}$ is applied by a block to the particle 1 , where $N_{L}>N_{0}$. 


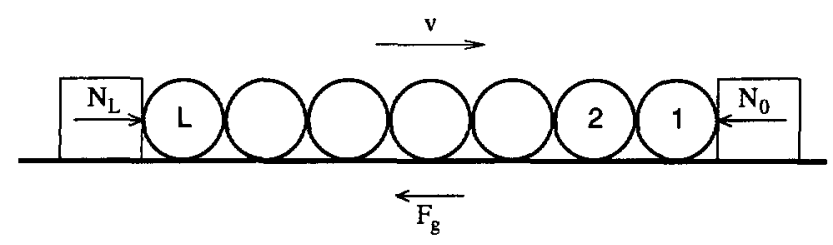

Fig. 1. - Geometry and boundary conditions of the model.

The mechanical parameters of the system are: particle-particle $(\mu)$, particle-plane $\left(\mu^{\prime}\right)$ and particle-block $\left(\mu^{\prime \prime}\right)$ coefficients of friction, driving and braking forces, the weight $P$ and the moment of inertia $I$ of each particle, and finally the total number of particles $L$. All quantities can, however, be normalized with respect to three basic units of the problem: the weight and the radius of one particle, and the acceleration of gravity; they are all set equal to unity. In the following, we will use only dimensionless quantities.

Each particle in the array has one rotational and two translational degrees of freedom. None of them is frozen in this model, so that particle-particle or particle-plane contacts may "open" during motion. Our simulations show, however, that starting the system with the closedcontact configuration (each particle in contact with its two neighbors and with the plane), all contacts are mechanically stable in time unless the value of the driving force $N_{L}$ exceeds a critical value $N_{\mathrm{c}}$ which is a function of the parameters. The degrees of freedom are thus reduced to only one translational for the whole array and one rotational per particle. In the simulations reported in this paper we have mostly used low values for the coefficients of friction in order to get a stable system for a wide range of driving force values.

It is important to note that the particle rotations in this one-dimensional system are fully frustrated. Indeed, every pair of neighboring particles and the plane form a loop of three solids mutually in contact. At least one of the three contacts has to be sliding. As the array is pushed, particles move and rotate and some interparticle or particle-plane contacts can be nonsliding. Moreover, during motion a NS contact may turn into a $\mathrm{S}$ contact, or vice versa. The correlations in the motions of particles, due to the multi-contact geometry and frustration of rotations, are so strong that collective dynamics should be expected.

We are interested in the evolution of the system and the behavior of the global arrayplane friction as a function of the local friction laws and the mechanical parameters. Let us first briefly introduce different friction laws which we have used and their implementation in numerical algorithms.

\section{Friction Laws}

A friction law is a relation between the relative tangential velocity $v^{t}$ and the friction force $T$ at a contact $(i j)$ between two solid bodies $i$ and $j$. We used three different friction laws, namely Coulomb's law and two variants of it.

3.1. CoulomB's Law (C1). - Figure 2a shows the graph of Coulomb's law. For a nonzero relative velocity $v^{\mathrm{t}}$, the friction force $T$ at a contact between two particles is equal to $-\operatorname{sgn}\left(v^{t}\right) \mu N$, where $\mu$ is the coefficient of friction. This corresponds to the two horizontal branches of Coulomb's graph, for which the friction force is opposite to the direction of relative motion. At $v^{\mathrm{t}}=0$ (NS contact), the friction force has a value in the interval $[-\mu N, \mu N]$. Note that $N$ is always a repulsive force and with a suitable choice of the local frame attached 

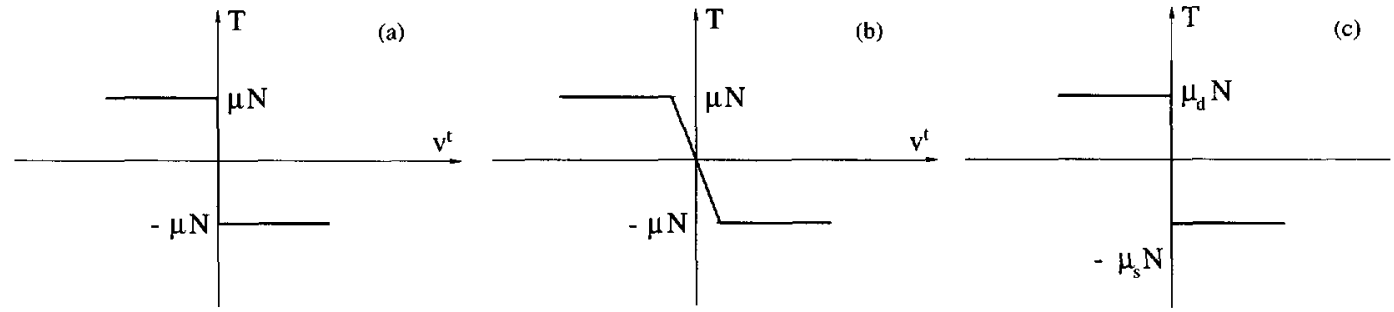

Fig. 2. - Graphs of friction laws, relating the relative tangential velocity $v^{t}$ and the friction force $T$ at a contact between two solid bodies: a) Coulomb's law used in CD simulations, b) regularized Coulomb's law used in molecular dynamic simulations, and c) Coulomb's law with a dynamic coefficient of friction $\mu_{\mathrm{d}}$ and a static coefficient of friction $\mu_{\mathrm{s}}$.

to a contact, it can be made positive. We remark also that at a NS contact the direction of the friction force can be either positive or negative. In the following, we will refer to this exact formulation of Coulomb's law as the C1 law.

Coulomb's law is nonsmooth in the sense that the two variables $v^{t}$ and $T$ belong to a continuous set of acceptable values which can not be represented as a function of either of the two variables $[10,11]$. The friction force at a NS contact is a reaction force: It is not given locally by the friction law itself, i.e. as a function of $v^{\mathrm{t}}$, but compensates the tangential component of all other forces acting on the two bodies in contact. In other words, friction at a NS contact involves inevitably the dynamics of the contacting bodies. The question is then how dynamics may be conveniently taken into account in the formulation of the friction law.

The point is that the distinction between NS and S contacts, suggested by the C1 law, is a kinematic definition of the state of a contact. If at a contact the relative tangent velocity $v^{\mathrm{t}}$ is zero but the relative tangent acceleration $\dot{v}^{\mathrm{t}}$ is not, then the contact cannot stay NS over whatever a small time interval. The friction force at such a contact is necessarily fully mobilized and we have $T=-\operatorname{sgn}\left(\dot{v}^{\mathrm{t}}\right) \mu N$, where $\dot{v}^{\mathrm{t}}$ is the relative tangential acceleration. A contact is NS in the dynamic sense only if we have both $v^{\mathrm{t}}=0$ and $\dot{v}^{\mathrm{t}}=0$. On the basis of this distinction between NS and S contacts in the dynamic sense, Coulomb's law takes the following general form for a contact $(i j)$ between two bodies $i$ and $j$ :

$$
\begin{aligned}
& v_{\imath \jmath}^{\mathrm{t}}=0 \text { and }\left\{\begin{array}{l}
\dot{v}_{\imath \jmath}^{\mathrm{t}}=0 \Rightarrow T_{\imath \jmath} \in\left[-\mu N_{\imath \jmath}, \mu N_{\imath \jmath}\right], \\
\dot{v}_{\imath \jmath}^{\mathrm{t}} \geq 0 \Rightarrow T_{\imath \jmath}=-\mu N_{\imath \jmath}, \\
\dot{v}_{\imath \jmath}^{\mathrm{t}} \leq 0 \Rightarrow T_{\imath \jmath}=\mu N_{\imath \jmath},
\end{array}\right. \\
& v_{\imath \jmath}^{\mathrm{t}}>0 \Rightarrow T_{\imath \jmath}=-\mu N_{\imath \jmath}, \\
& v_{\imath \jmath}^{\mathrm{t}}<0 \Rightarrow T_{\imath \jmath}=\mu N_{\imath \jmath} .
\end{aligned}
$$

Coulomb's law is written here as a set of possible alternatives, each of them involving one equation and one inequality. The important point is that all of these equations contain only dynamic variables (force or relative acceleration). Consider, for instance, the first case where $v^{\mathrm{t}}=0$ and $\dot{v}^{\mathrm{t}}=0$. The latter can be supplemented to the equations of dynamics. However, the calculated friction force $T$ should satisfy the corresponding inequality $T \in[-\mu N, \mu N]$. If this condition is satisfied, then the contact is NS in the dynamic sense, i.e. $\dot{v}^{\mathrm{t}}=0$, and we have got the solution for all dynamic variables. Otherwise, we should pass to the next alternative. All alternatives are to be tested in this way until the solution is found. For more details concerning the implementation of this procedure, see Appendix A. 
3.2. A Regularized Coulomb's LaW (RC1). - A different method widely used in simulation of dense granular systems, the molecular dynamics (MD) method [13], cannot handle the exact form of Coulomb's law. In MD simulations, Newton's equations of motion are integrated numerically, so that the method requires specification of the forces acting between grains. The forces thus have to be functions of the particle positions and velocities.

Here, we are mainly concerned with the tangential force. The details concerning the normal force can be found in Appendix B. Since Coulomb's law cannot be implemented directly, it has to be regularized for $v^{\mathbf{t}} \approx 0$. One widely used choice [15-21] to achieve this is to approximate the tangential force $T$ by the function

$$
T=-\operatorname{sgn}\left(v^{\mathrm{t}}\right) \min \left\{\gamma_{\mathrm{s}}\left|v^{\mathrm{t}}\right|, \mu N\right\}
$$

where $\gamma_{s}$ is an adjustable parameter (see Fig. 2b). The higher $\gamma_{s}$, the closer the regularized law is to the nonsmooth one. We emphasize that this viscous friction law has no physical justification. It is just a convenient approximation whose consequences in numerical simulations of granular systems so far have only been checked for the case of the binary impact of particles [14]. For this case it was found that $\gamma_{\mathrm{s}}$ should be as large as possible to achieve the results expected for the Coulomb friction law. The 1D model with its simple geometry provides a good model system to investigate the physical outcome of this contact law in the case of long-lasting contacts.

In a densely packed system there is a great number of NS contacts. With the regularized Coulomb's law, which we will refer to as the RC1 law from now on, we cannot get a true NS contact, i.e. $v^{\mathrm{t}}=0$, except in the improbable case $T=0$. Deviation from a true NS contact increases with the normal force and the coefficient of friction, since the Coulomb range $[-\mu N, \mu N]$ increases then. This error gives rise also to an error in dissipation. Whereas a true NS contact is non-dissipative, the RC1 law gives a dissipation rate equal to $\gamma_{\mathrm{s}}\left(v^{\mathrm{t}}\right)^{2}$ at a NS contact [22].

3.3. Coulomb's Law with Two Coefficients (C2). - It is a well-known property of most materials that the coefficient of friction at the sliding threshold, i.e. the static coefficient $\mu_{\mathrm{s}}$, is higher than that during sliding, i.e. the dynamic coefficient $\mu_{\mathrm{d}}$. This property in association with a sufficiently elastic driving mechanism gives rise to stick-slip motion $[9,24]$. Figure 2c shows the graph of a Coulomb law with two coefficients of friction. We will refer to this law as the $\mathrm{C} 2$ law from now on. The Coulomb range of the friction force in the C2 law for a NS contact is $\left[-\mu_{\mathrm{s}} N, \mu_{\mathrm{s}} N\right]$.

As in the case of the $\mathrm{C} 1$ law, the $\mathrm{C} 2$ law has to be interpreted in the dynamic sense. We may again use the $\mathrm{CD}$ method to simulate this nonsmooth law, taking numerically care of the static Coulomb range at NS contacts. In fact, the iteration scheme for searching the contact states here is more complicated. Our simulations show that the solution with the C2 law is not unique.

\section{Evolution}

The initial state is completely given by the angular velocities of particles $\omega(i)$ and the linear velocity $v$ of the array. With a random distribution of velocities we generally get a state where all contacts are sliding.

The evolution of the system as a whole can be described by the variation of the linear acceleration $\dot{v}$ of the array, or equivalently of the global friction $F_{\mathrm{g}}$, defined as the sum of friction forces exerted by the plane on particles. The two global dynamic variables $\dot{v}$ and $F_{\mathrm{g}}$ are related together by the equation of motion of the center of mass of the system,

$$
N_{L}-N_{0}-F_{\mathrm{g}}=m_{\mathrm{g}} \dot{v},
$$




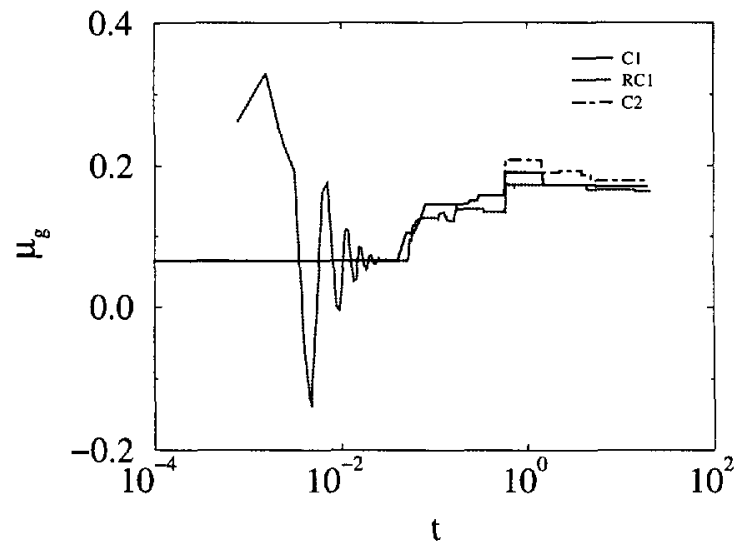

Fig. 3. - Typical evolution of the global coefficient of friction $\mu_{\mathrm{g}}$ for an array of 10 particles with the $\mathrm{C} 1, \mathrm{C} 2$ and $\mathrm{RC} 1$ friction laws as a function of the dimensionless time $t$ (see text) for the same parameters and initial conditions. Parameters are $N_{L}=2.5, N_{0}=0.01, \mu=0.1, \mu^{\prime}=0.2$ and $\mu^{\prime \prime}=0.001$ for the $\mathrm{C} 1$ and $\mathrm{RC} 1$ laws. For the C2 law we have $\mu_{\mathrm{s}}=0.3, \mu_{\mathrm{s}}^{\prime}=0.6, \mu_{\mathrm{d}}=0.1, \mu_{\mathrm{d}}^{\prime}=0.2$ and $\mu^{\prime \prime}=0.001$.

where $m_{\mathrm{g}}=L$ is the total mass of the system. A global coefficient of friction $\mu_{\mathrm{g}}$ can be defined for the array-plane contact by $\mu_{\mathrm{g}}=F_{\mathrm{g}} / L$.

Figure 3 shows a typical evolution of $\mu_{\mathrm{g}}$ for an array of 10 particles with the $\mathrm{C} 1, \mathrm{C} 2$ and $\mathrm{RC} 1$ friction laws for the same common parameters and initial conditions. The $\mathrm{C} 1$ law (simulated by the CD method) gives a piecewise constant path. Each jump is a discontinuous variation of all forces and accelerations as a consequence of changing of one contact state in the system. The MD method gives a completely different path. The oscillations at very short time are clearly a consequence of contact elasticity. If the normal restoring force and the damping are weaker, these oscillations dominate the path even at longer times. But as soon as the oscillations settle down, friction (the RC1 law) takes over and the path shows a behavior similar to the C1 path. For the C2 law, we have again a stepwise path which differs partially from the C1 path.

In all cases, the evolution of the system ends in a steady state where the dynamic variables (forces and accelerations), and $\mu_{\mathrm{g}}$ as a result, stay constant in time. Physically, this corresponds to the state where the relative tangential velocity and the relative tangential acceleration at every contact are of the same sign, or both are equal to zero [23]. In Contact Dynamics simulations (with the $\mathrm{C} 1$ law) this latter rigorous criterion is used and the steady state is reached whatever the initial conditions. In molecular dynamics simulations (with the $\mathrm{RC1}$ law) the steady state is sometimes not reached in this rigorous sense but only as a state where the dynamic variables stay constant within some precision, although contact states continue to change from time to time. For a reliable identification of the steady state in MD simulations, both the exact condition from CD, as well as some precision limit on the fluctuations of the dynamic variables should be checked. These fluctuations, however, do not influence considerably the average steady-state value of $\mu_{\mathrm{g}}$ with the RC1 law, which is always very close to that obtained with the $\mathrm{C} 1$ law. The discrepancy among the steady-state values of $\mu_{\mathrm{g}}$, or other dynamical variables of the system, are more pronounced for the $\mathrm{C} 2$ law on one hand and the two other laws on the other hand.

Figure $4 \mathrm{a}$ shows the evolution of $\mu_{\mathrm{g}}$ simulated by $\mathrm{CD}$ with the $\mathrm{C} 1$ law for different initial conditions: The steady state is independent of initial conditions even though the number 

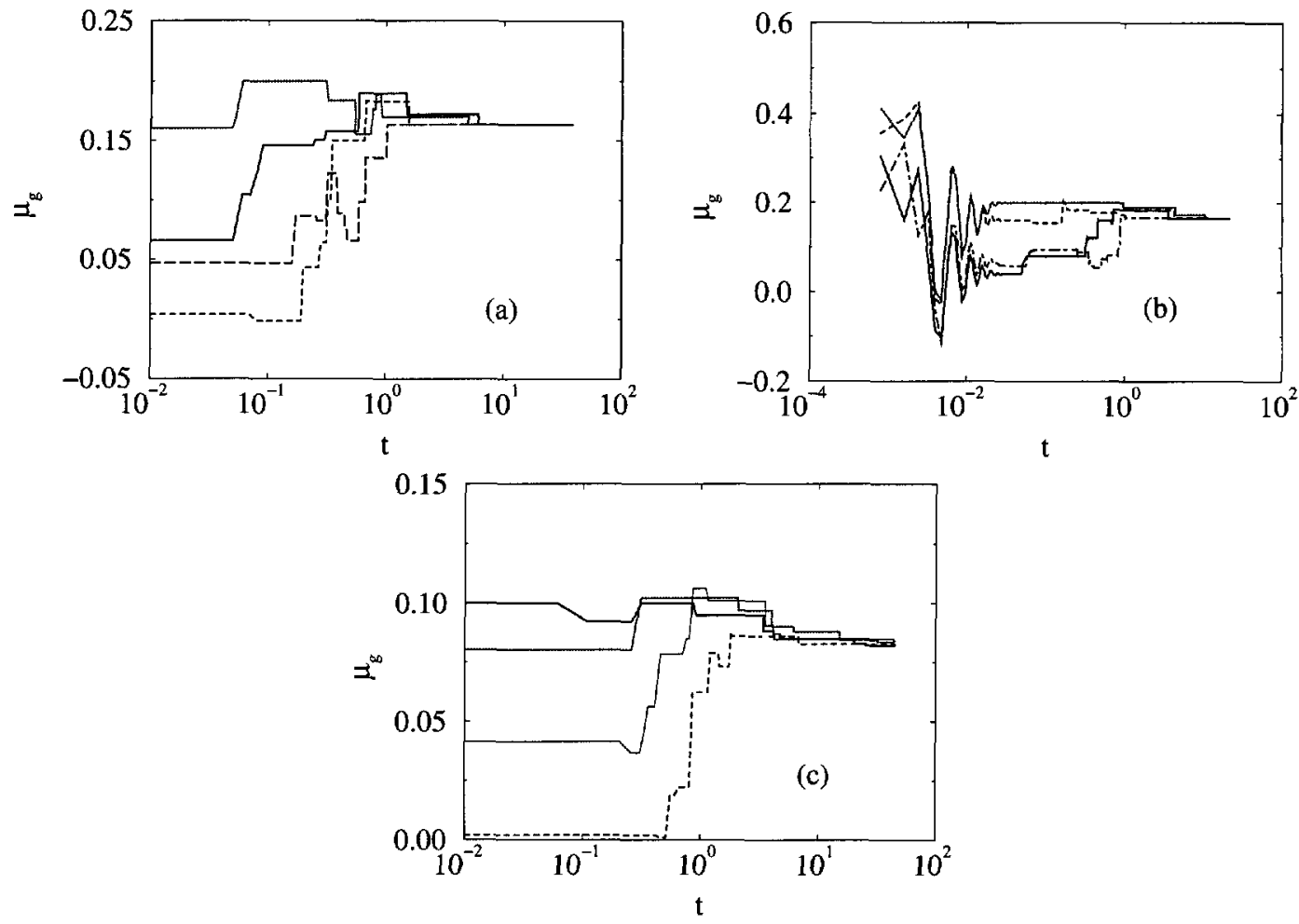

Fig. 4. - Evolution of the global coefficient of friction $\mu_{\mathrm{g}}$ simulated by (a) the CD method using the C1 law, (b) the MD method with the RC1 law, and (c) by the CD method with the C2 law, as a function of the dimensionless time $t$ for different initial conditions. Parameters are the same as in Figure 3. Note that in (c) we have $\alpha=\mu_{s} / \mu_{d}=\mu_{s}^{\prime} / \mu_{d}^{\prime}=3$.

of possible transients grows exponentially with the system size. This independence of the steady state from the initial conditions is conceptually important. It means that the steadystate value of $\mu_{\mathrm{g}}$ is an intrinsic quantity depending only on system parameters and not on the preparation of the system. This is one step towards the definition of a Coulombian friction force for the array as a whole.

For most initial conditions, $\mu_{\mathrm{g}}$ is initially weak. Then it grows, goes through a maximum, and finally reaches its steady state value. At the beginning, for a random distribution of rotation velocities, the relative velocities at particle-plane contacts are randomly negative or positive. So, at almost half of the particle-plane contacts the friction force is initially oriented in the direction of motion of the array, whereas the other half is directed opposite to it. For this reason $\mu_{\mathrm{g}}$, which is given by the sum of the particle-plane friction forces, is initially weak. As the angular velocities of particles and the linear velocity of the array evolve, the friction forces exerted by the plane on particles (at S but also at NS particle-plane contacts) gradually become opposite to the direction of motion of the array and $\mu_{\mathrm{g}}$ increases correspondingly.

For later times, $\mu_{\mathrm{g}}$ decreases again. This is due to the emergence of stable NS contacts and can be understood in the following way: $F_{\mathrm{g}}$ in (3) may be interpreted alternatively as sum of all forces exerted by the plane on the array of particles or as dissipation rate/ $v$. The NS particle-plane contacts contribute only in the first picture, whereas in the second only sliding, 
i.e. dissipative contacts count (including those among particles). At late times the number of sliding contacts decreases, and hence $\mu_{\mathrm{g}}$ decreases as well.

More generally, since the steady state with the $\mathrm{C} 1$ and $\mathrm{RC} 1$ laws is independent of initial conditions, the question is how the memory of initial conditions is lost. At the beginning all contacts are sliding. The appearance of the first NS contacts is directly dictated by the initial angular velocities of the particles. However, they are generally misplaced as compared to the positions of NS contacts in the steady state. So, most of NS contacts appearing before the maximum are metastable and soon turn again into $\mathrm{S}$ contacts. The dominant process before the maximum is thus $\mathrm{S} \rightarrow \mathrm{NS} \rightarrow \mathrm{S}$. This is what clears the memory of initial velocities. In fact, in the transition $\mathrm{S} \rightarrow \mathrm{NS}$ the initial relative velocity is lost. Then, the value and the direction of the relative velocity in the new transition NS $\rightarrow \mathrm{S}$ is dictated by that of the relative acceleration, which is in turn related to dynamics of the whole array and its boundary conditions. After the maximum, the dominant process is S $\rightarrow$ NS. The NS contacts are then generally stable and will gradually shape the organization of rotations in the steady state.

In Figure $4 \mathrm{~b}$ we have shown the evolution of $\mu_{\mathrm{g}}$ simulated by MD with the RC1 law for different initial conditions. Here also the steady state is independent of initial conditions. In the framework of the MD approach there is no straightforward way to explain how the system may forget its initial state. But independence of the steady state with respect to initial conditions shows that, as soon as initial elastic oscillations are damped away, a regularized friction law can produce results similar to those of the nonsmooth law.

In the case of the $\mathrm{C} 2$ law, the steady state depends on initial conditions, as shown in Figure 4c. To understand this point, let us consider the extreme case where $\alpha=\mu_{\mathrm{s}} / \mu_{\mathrm{d}}$ tends to infinity (here by $\mu_{\mathrm{s}}$ or $\mu_{\mathrm{d}}$ we mean coefficients of friction at any particle-particle or particle-plane contact). Then a contact where $v^{\text {t }}$ becomes zero necessarily remains NS, since the static friction can be mobilized to infinity. In this way, the first NS contacts which depend essentially on the initial angular velocities of particles and the linear velocity of the array will be stable, whereas in the case of the C1 law most of the NS contacts appearing in the beginning are unstable. For lower values of $\alpha$ a given NS contact has more chance to turn $\mathrm{S}$ and we should thus expect less dependence on initial conditions. Figure $4 \mathrm{c}$ shows that even for $\alpha=3$, the steady-state values of $\mu_{\mathrm{g}}$ are very close to each other.

\section{Collective Modes}

The most distinctive feature of the steady state is the beautiful organization of particle rotations along the system [8]. Figure 5 shows the angular accelerations $\dot{\omega}(i)$ of particles as a function of their position in the array, as simulated using the C1 law. In this figure we can distinguish three spatial domains with collective rotation modes. In the first domain, next to the braking block, all particles roll on the plane, i.e. $\dot{\omega}(i)=\dot{v}$ (mode 1 ). In the next domain particles rotate and slide on the plane, with a decreasing angular velocity along the array (mode 2 ). In the last domain, next to the driving block, all particles are counter-rotating, i.e. $\dot{\omega}(i)+\dot{\omega}(i+1)=0$ (mode 3). A particle in mode 1 or 3 has at least one NS contact, whereas in mode 2 all contacts of each particle are sliding.

Modes 1 and 2 extend over coherence lengths $L_{1}^{\mathrm{c}}$ and $L_{2}^{\mathrm{c}}$ which are functions of the parameters and the linear acceleration of the array [8]. These lengths can be significantly larger than the particle diameters, but do not depend on the system size. They characterize the system on a mesoscopic scale. Mode 3 fills the rest of the system and is only present if $L>L_{1}^{\mathrm{c}}+L_{2}^{\mathrm{c}}$.

$L_{2}^{\mathrm{c}}$ increases with $\dot{v}$, whereas $L_{1}^{\mathrm{c}}$ and $L_{1}^{\mathrm{c}}+L_{2}^{\mathrm{c}}$ decrease. Mode 2 is a direct consequence of the accelerated motion of the array. A particular case is the limit of constant velocity, where $\dot{v}=0$. Then, mode 2 disappears from the array, i.e. $L_{2}^{\mathrm{c}}=0$, and mode 1 takes its largest 


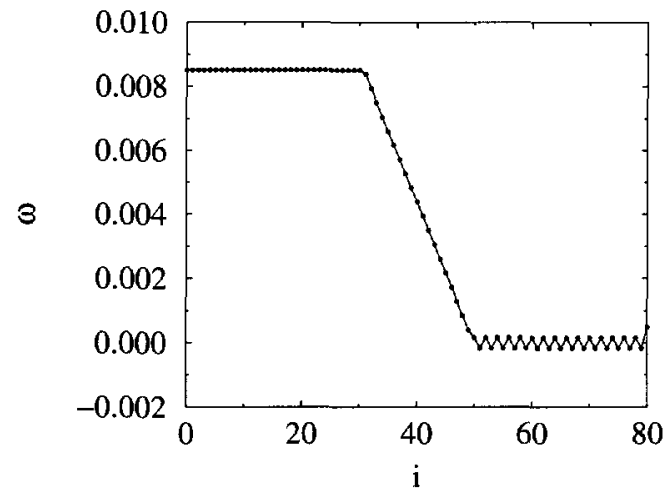

Fig. 5. - Dimensionless steady-state angular accelerations $\dot{\omega}(i)$ of particles as a function of their positions $i$ in an array of 80 particles, as simulated with the C1 law. Parameters are $N_{L}=1.4$, $N_{0}=0.01, \mu=0.06, \mu^{\prime}=0.1$ and $\mu^{\prime \prime}=0.001$. The acceleration shown on abscisse 0 is the dimensionless linear acceleration of the array as a whole: $\dot{\omega}(0)=\dot{v}$.
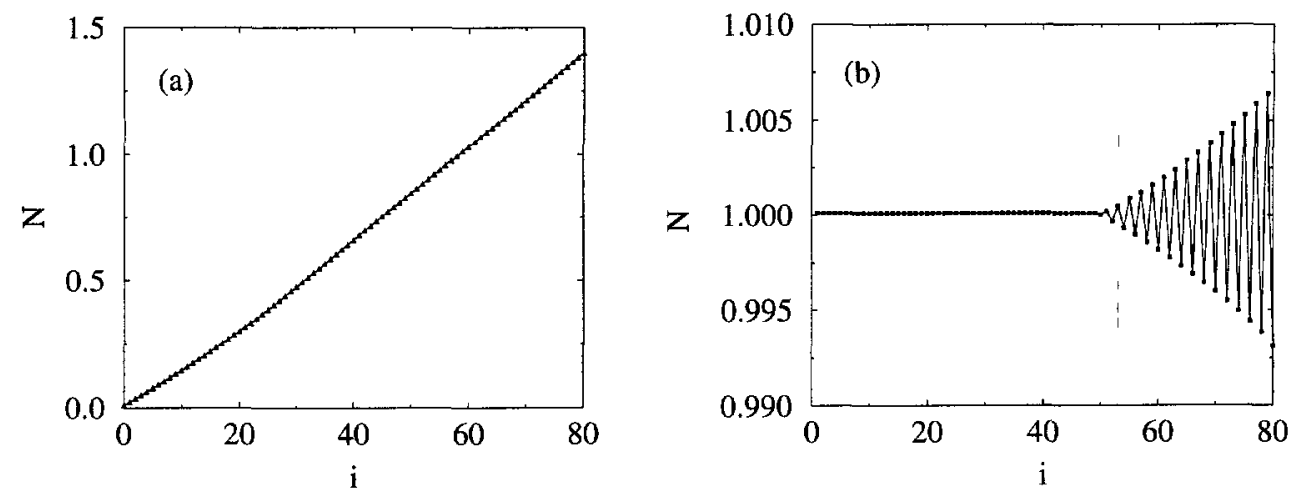

Fig. 6. - Particle-particle and particle-plane dimensionless steady-state normal forces $N(i)$ (a) and $N^{\prime}(i)$ (b), respectively, as a function of the positions $i$ of contacts in an array of 80 particles. The contact number 0 is the one between the first particle and the braking block. Parameters are the same as in Figure 5.

coherence length for a given set of parameters [8]. For $\mu \ll \mu^{\prime}$ all particles are in mode 1, i.e. $L_{1}^{\mathrm{c}}>L$, whereas for $\mu^{\prime} \ll \mu$ all particles are in mode 3 , because $L_{1}^{\mathrm{c}}$ becomes smaller than the diameter of a particle.

Our simulations with the RC1 law (by MD) give the three modes in good agreement with the results obtained with the $\mathrm{C} 1$ law (by $\mathrm{CD}$ ). With the $\mathrm{C} 2$ law we may get a large variety of steady states with different values of $L_{1}^{\mathrm{c}}$ and $L_{2}^{\mathrm{c}}$, depending on the initial state. The major effect of the static coefficient of friction is to reinforce NS contacts. In this way, mode 2 may disappear completely from the array for sufficiently large $\alpha$. The collective modes have also been observed in experiments with a linear array of cylinders [24].

In Figures 6 and 7 , we have plotted the contact forces for the system of Figure 5 . In mode 1 the friction force at particle-plane contacts is only partially mobilized. The degree of mobilization, which can be defined by $\eta=|T| /(\mu N)$, increases along the first domain 

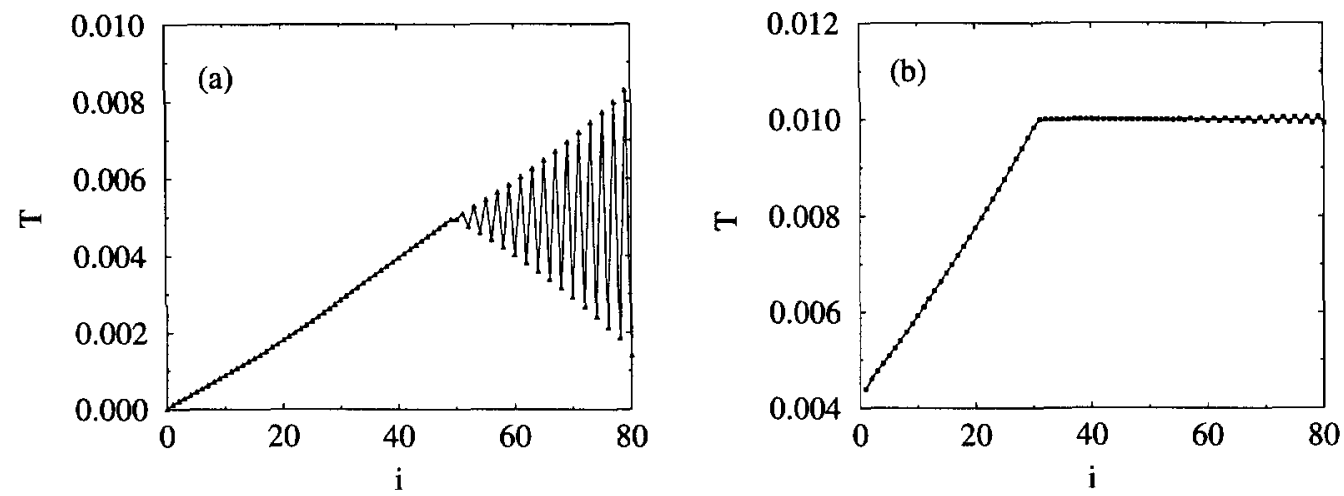

Fig. 7. - Particle-particle and particle-plane dimensionless steady-state friction forces $T(i)(a)$ and $T^{\prime}(i)(\mathrm{b})$, respectively, as a function of the positions $i$ of contacts in an array of 80 particles. Parameters are the same as in Figure 5.

(exponentially, according to the theoretical analysis of [8]). Only for the first particle of mode 2 the value of $\eta$ at the particle-plane contact reaches its maximum $\eta=1$. This determines $L_{1}^{\mathrm{c}}$.

The situation is different for mode 2, where all contacts are sliding. At every contact of each particle in this mode, the friction force is proportional to the normal force. The particleparticle friction increases along the array, while the particle-plane friction remains constant. As a result, the angular acceleration decreases along this domain in absolute value and changes sign for the first particle in mode 3 .

In mode 3, the particle-particle contacts are NS. The friction force at such contacts is partially mobilized. It oscillates with exponentially varying envelopes. However, in contrast to mode 1, $\eta$ stays always lower than unity, so that the length of mode 3 is theoretically infinite. The limit on the effective length of this domain comes from the possibility of the rising of a particle (the one in contact with the driving block or the one before) when the particle-particle friction force is too high. This is, in fact, the limit of the model for a given set of parameters.

\section{Steady-State Friction}

In this section we discuss how the global steady-state friction depends on the driving force, on the length of the array and on local coefficients of friction.

6.1. DePendence on Driving Force. - We showed that the steady-state coefficient of friction, $\mu_{\mathrm{g}}$, is a good parameter for characterizing the "macroscopic" friction of the array as a whole, since it is independent of initial conditions for the $\mathrm{C} 1$ and RC1 laws. Nevertheless, contrary to a "Coulombian" coefficient of friction, $\mu_{\mathrm{g}}$ (in the steady state) is an increasing function of the driving force $N_{L}$ and saturates at the value of the particle-plane coefficient of friction for high enough values of $N_{L}$ [25]. This is shown in Figure 8.

The motion of the array as a whole is thus governed by two equations. One of them is the equation of motion ( 3 ) of the center of mass of the system; the other one is the dependence of $F_{\mathrm{g}}$ on the driving force or, equivalently, on the linear acceleration $\dot{v}$ of the array, which can be written as a general equation:

$$
F_{\mathrm{g}}=F_{\mathrm{g}}(\dot{v}, \Omega),
$$




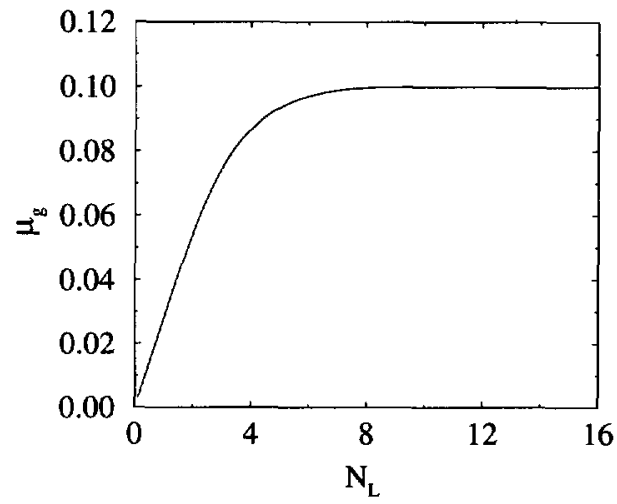

Fig. 8. - Steady-state global coefficient of friction $\mu_{\mathrm{g}}$ as a function of the dimensionless driving force $N_{L}$ for an array of 30 particles as simulated with the C1 law. Parameters are $N_{0}=0.01, \mu=0.04$, $\mu^{\prime}=0.1$ and $\mu^{\prime \prime}=0.001$.

where $\Omega$ stands for all mechanical parameters of the system. If $F_{\mathrm{g}}$ were a linear function of $\dot{v}$, we might separate the term proportional to $\dot{v}$ and move it to the right hand side of $(3)$. We would then get an "effective" inertia for the system higher than its mass. However, as Figure 8 shows, dependence on $\dot{v}$ (or $N_{L}$ ) is not generally linear. One may then resort to an infinitesimal analysis in order to separate the contribution of $F_{\mathrm{g}}$ to an effective inertia in a unique way. In this way, we introduce the following Legendre transform of $F_{\mathrm{g}}$ as a function of $\dot{v}$ :

$$
\begin{aligned}
a & =\partial F_{\mathrm{g}} / \partial \dot{v} \\
b & =F_{\mathrm{g}}-a \dot{v} .
\end{aligned}
$$

By introducing the new variables into (3), we get the following equation for the center of mass of the system:

$$
N_{L}-N_{0}-F_{\mathrm{e}}=m_{\mathrm{e}} \dot{v}
$$

where $F_{e}=b$ and $m_{e}=m_{\mathrm{g}}+a . F_{\mathrm{e}}$ and $m_{\mathrm{e}}$ are the effective friction force and the effective inertia, respectively. We may also define an effective coefficient of friction by $\mu_{\mathrm{e}}=F_{\mathrm{e}} / \mathrm{m}_{\mathrm{g}}$.

The effective quantities $\mu_{\mathrm{e}}$ and $m_{\mathrm{e}}$ vary with $\dot{v}$, as shown in Figures 9a, b. The two plots, obtained by the CD method, are piecewisely constant functions composed of two types of discontinuous changes: steps and "spikes". These details were not discussed in [8], since there they were not seen due to a lower resolution. At low enough values of $\dot{v}$, all particles are in mode 1. The effective inertia is very high (almost six times the total mass $m_{\mathrm{g}}=30$ of the system in these simulations!), whereas the effective coefficient of friction is very low. As soon as the particle $i=L$ enters mode 2 for some value of $\dot{v}, m_{\mathrm{e}}$ jumps down, while $\mu_{\mathrm{e}}$ jumps up. As $\dot{v}$ increases, more and more particles enter modes 2 and 3 and the contact states change. Each changing of a contact state gives rise to a step.

The spikes appear every time the direction of angular accelerations of particles in domain 3 is reversed. Each spike has a finite width. After each spike the values of $m_{\mathrm{e}}$ and $\mu_{\mathrm{e}}$ come back almost to the same level as before the spike with a reversed direction of particle rotations in domain 3 .

At high enough values of $\dot{v}$ all particles are in mode 3 . The contact states will no more change with increasing $\dot{v}$, so that the effective quantities stay constant as a function of $\dot{v}$. Then, $m_{\mathrm{e}}=m_{\mathrm{g}}$ and $\mu_{\mathrm{e}}=\mu_{\mathrm{g}} \approx \mu^{\prime}$. 

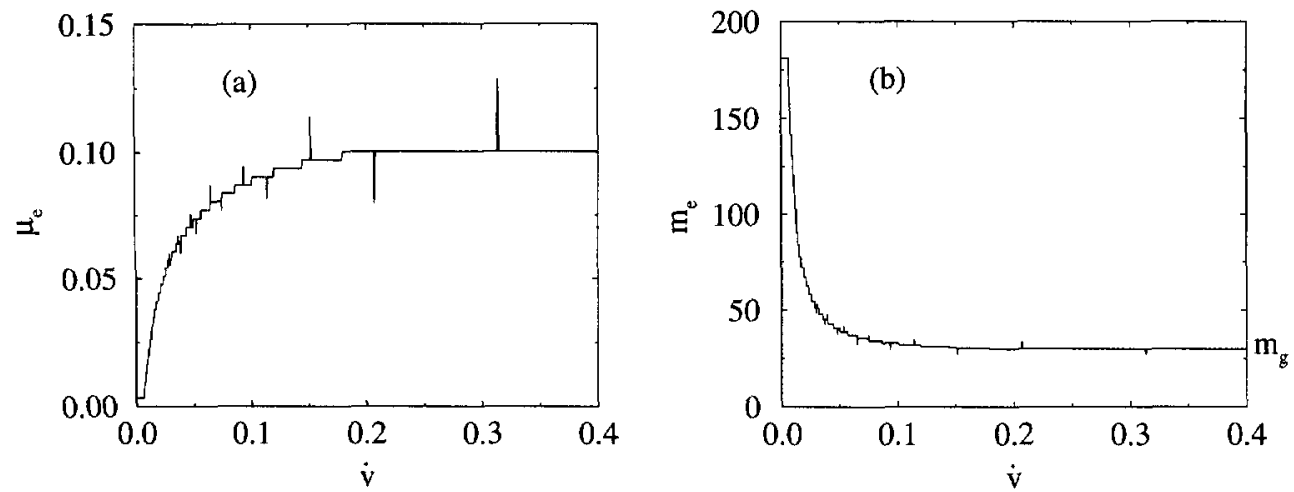

Fig. 9. - Effective coefficient of friction $\mu_{\mathrm{e}}$ (a) and effective inertia $m_{\mathrm{e}}$ (b) as a function of the dimensionless linear acceleration $\dot{v}$ of an array of 30 particles. Parameters are the same as in Figure 8.

Physically, the effective inertia of the system is a consequence of particle rotations on the plane. The friction force mobilized at a particle-plane contact does not totally resist the motion of the array. On the contrary, it applies a positive moment on the particle that contributes to its rolling on the plane. The real resistance to rolling on the plane is, in fact, localized at particle-particle sliding contacts. In mode 1, it increases with the normal particle-particle force proportionally to the driving force. That is why the dissipative resistance to rolling appears as an additional inertia whose expression depends on the particle-particle coefficient of friction. In the same way, the effective friction is a purely dissipative resistance to the translational motion of the particle. The analytic expressions of the effective quantities in mode 1 (which gives the main contribution) are given by [8]:

$$
\begin{aligned}
& m_{\mathrm{e}}^{(1)}=\frac{1+I}{2 \mu}\left[\left(\frac{1+\mu}{1-\mu}\right)^{L_{1}}-1\right], \\
& \mu_{\mathrm{e}}^{(1)}=\frac{1}{L_{1}}\left[\left(\frac{1+\mu}{1-\mu}\right)^{L_{1}}-1\right] N_{0},
\end{aligned}
$$

where $L_{1}$ is the length of domain 1 . We see that the two quantities increase with $\mu$. In the case of mode 2, the before-mentioned mechanism is still partially present, i.e. the particleplane friction force contributes to rotation on the plane. All contacts resist to rotation and to translation on the plane at the same time. We get the following expressions:

$$
\begin{aligned}
m_{\mathrm{e}}^{(2)} & =\frac{1}{1-\mu \mu^{\prime}} L_{2}, \\
\mu_{\mathrm{e}}^{(2)} & =\frac{\mu^{\prime}}{1-\mu \mu^{\prime}},
\end{aligned}
$$

where $L_{2}$ is the length of domain 2. Finally, in mode 3 the effective inertia oscillates around the total mass of the array, whereas the effective friction oscillates around the value of the global friction [8]. However, the friction force at the contact of each particle in mode 3 rotating in the positive direction (the direction of motion of the array) with the plane resists less to motion than that of a particle in mode 3 rotating in the negative direction. When the angular accelerations of particles in domain 3 are zero, the friction force at particle-plane contacts is 


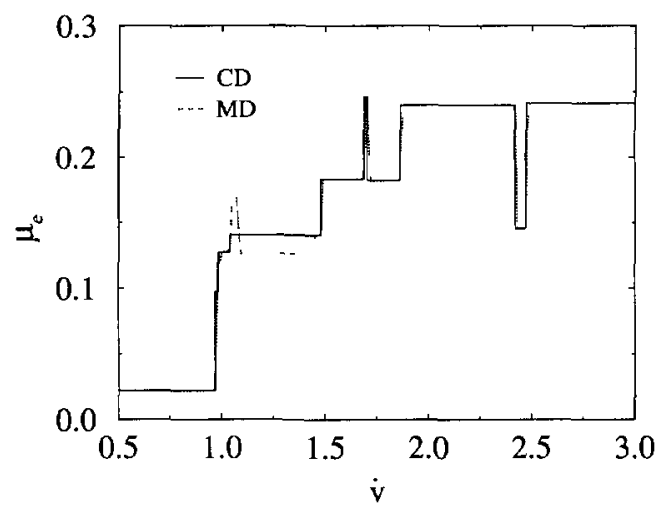

Fig. 10. - Effective dimensionless coefficient of friction $\mu_{\mathrm{e}}$ as a function of the dimensionless linear acceleration $\dot{v}$ of an array of 5 particles as simulated by CD and MD methods. Parameters are $N_{L}=0.9, N_{0}=0.01, \mu=\mu^{\prime}=0.3$ and $\mu^{\prime \prime}=0.01$.

only resisting the motion and the contact with the pushing block is NS. This is the reason for the appearance of spikes every time the rotation of particles in domain 3 changes direction. A spike has a finite width since the friction force at a NS particle-block contact can be mobilized within the Coulomb range $\left[-\mu^{\prime \prime} N_{L}, \mu^{\prime \prime} N_{L}\right]$ in such a way to stop the rotations of particles in domain 3. That is why the amplitude and the width of spikes increase linearly with the driving force. Moreover, in the case where $\mu^{\prime \prime}=0$ the spikes disappear from the plot. We see that Figure $9 \mathrm{a}$ (or Fig. 9b) contains all the information concerning the evolution of contact states as a function of $\dot{v}$ : The steps correspond to the changing of particle-particle or particle-plane contact states and the spikes to the changing of the state of the contact with the pushing block.

It is instructive here to return to the comparison between the $\mathrm{C} 1$ and $\mathrm{RC} 1$ laws. With the RC1 law we get essentially the same plots for the effective quantities as a function of $\dot{v}$ as with $\mathrm{C} 1$. The reason is that only the steady-state friction is considered here and we have seen that the elastic oscillations, observed in Figure $4 \mathrm{~b}$ during the evolution of the system, do not influence the steady state. Nevertheless, in some cases discrepancies appear also in the steady state. One example is given in Figure 10 where we plot the effective coefficient of friction as a function of $\dot{v}$ for a system of 5 particles as simulated by CD and MD separately. The agreement is quite good everywhere except around $\dot{v}=1.05$. The particle rotations in the system are shown in Figure 11 for $\dot{v}=1.05$. What is particular with this configuration is that the angular accelerations of the particles in mode 1 and mode 3 have the same absolute value. Hence, the three contacts of particle 3 are NS, and, as we know, the difference between the $\mathrm{C} 1$ and $\mathrm{RC} 1$ laws is at such NS contacts. If there are many NS contacts in a (less frustrated) system, we should therefore expect such discrepancies to be enhanced.

Let us now consider the limit of constant velocity or static equilibrium, $\dot{v}=0$. Since the global coefficient of friction increases with $\dot{v}$, its minimum is reached at $\dot{v}=0$. All particles are in mode 1 if $L<L_{1}^{\mathrm{c}}$. In this case, the global coefficient of friction is equal to $\mu_{\mathrm{g}}=\mu_{\mathrm{e}}^{(1)}$, whose expression is given by (8). If the C2 law is used, we have a global static coefficient of friction $\mu_{\mathrm{g}}^{\mathrm{s}}$ and a global dynamic one $\mu_{\mathrm{g}}^{\mathrm{d}}$. The first one is given by (8) by replacing the static particleparticle coefficient of friction $\mu_{\mathrm{s}}$ with $\mu$. The second one is given by the same expression with $\mu=\mu_{\mathrm{d}}$. Note that the difference $\mu_{\mathrm{g}}^{\mathrm{s}}-\mu_{\mathrm{g}}^{\mathrm{d}}$ in this regime increases exponentially with $L_{1}$, so that the static global coefficient of friction can be much higher than the dynamic one. Each particle added to the array increases the difference between the two coefficients. In particular, 


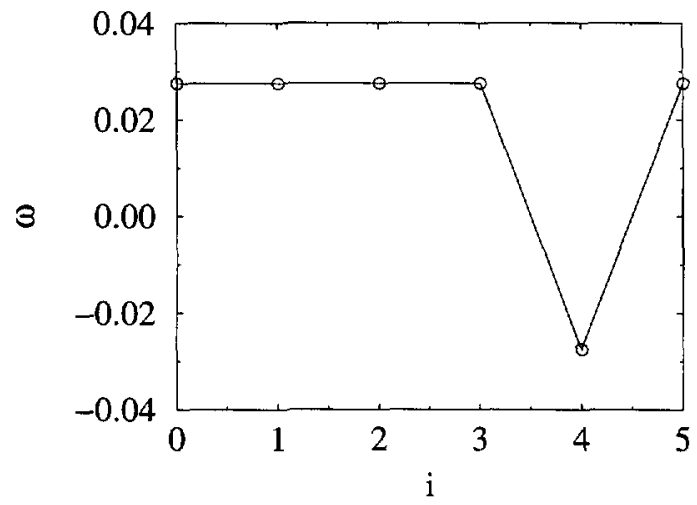

Fig. 11. - Dimensionless steady-state angular accelerations of particles in the array of Figure 10 at $\dot{v}=1.05$. The acceleration shown on abscisse 0 is the dimensionless linear acceleration of the array.

if the system is at incipient sliding, the addition of one particle can immobilize the system! This is because the motion of the array implies the mobilization of friction at all interparticle contacts in the system up to the static limit (given by the product of the coefficient of friction and the normal force), while the normal interparticle force increases exponentially along the array in mode 1.

If $L>L_{1}^{\mathrm{c}}$, there are $L-L_{1}^{\mathrm{c}}$ particles in mode 3 , since as mentioned before mode 2 disappears in the static limit. The static and dynamic coefficients of friction of mode 3 are approximately equal to $\mu_{\mathrm{s}}^{\prime}$ and $\mu_{\mathrm{d}}^{\prime}$, respectively. These coefficients are those of particle-plane contacts. $\mu_{\mathrm{g}}$ is the mean value of the global coefficients of friction of modes 1 and 3 weighted by their respective lengths:

$$
\mu_{\mathrm{g}}=\frac{1}{L}\left\{\left(\frac{1+\mu}{1-\mu}\right)^{L_{\mathrm{i}}^{\mathrm{c}}}-1\right\} N_{0}+\frac{L-L_{1}^{\mathrm{c}}}{L} \mu^{\prime} .
$$

This expression gives $\mu_{\mathrm{g}}^{\mathrm{s}}$ if $\mu=\mu_{\mathrm{s}}$ and $\mu^{\prime}=\mu_{\mathrm{s}}^{\prime}$. It gives $\mu_{\mathrm{g}}^{\mathrm{d}}$ if $\mu=\mu_{\mathrm{d}}$ and $\mu^{\prime}=\mu_{\mathrm{d}}^{\prime}$. As $L$ increases, $\mu_{\mathrm{g}}^{\mathrm{s}}$ approaches $\mu_{\mathrm{s}}^{\prime}$, and $\mu_{\mathrm{g}}^{\mathrm{d}}$ approaches $\mu_{\mathrm{d}}^{\prime}$.

6.2. Dependence on Number of Particles. - Now we show that $\mu_{\mathrm{g}}$ in the steady state depends on the number of particles, $L$. This means that the global friction violates another important property of Coulomb's friction law, as $L$ is the "apparent contact area".

Let us consider first an array totally in mode 1 . As long as all particles are in mode 1 , the effective coefficient of friction is the one given by (8). $\mu_{\mathrm{g}}$ for this mode increases very rapidly with $L$. However, at first order in $\mu$ the effective inertia is equal to $(1+I) L_{1}$ and the effective coefficient of friction is given by $2 \mu N_{0}$, which is independent of $L$. We recall that $\mu$ should be sufficiently small if the array is totally in mode 1 . On the other hand, if we define $\mu_{\mathrm{e}}$ by $F_{\mathrm{e}} / m_{\mathrm{e}}$ (and not by $F_{\mathrm{e}} / m_{\mathrm{g}}$ ), then its expression is independent of $L_{1}$. This definition provides a coefficient of friction for mode 1 which is independent of $L$ at all orders of $\mu$. But, this is not a common definition of the macroscopic coefficient of friction.

Equation (10) shows that $\mu_{\mathrm{e}}$ of mode 2 does not depend on the number of particles in this mode. This is also true for mode 3 up to small oscillations around $\mu_{\mathrm{e}}^{(3)}=\mu^{\prime}$, which is the effective coefficient of friction for this mode. On the other hand, the length of the first domain does not change with that of the array, since the new particles added to the array will find themselves in mode 3 or, if mode 3 is absent, in mode 2 . In this way, since the length of domains 2 and 3 , which have higher global coefficient of friction than the first domain, increases 

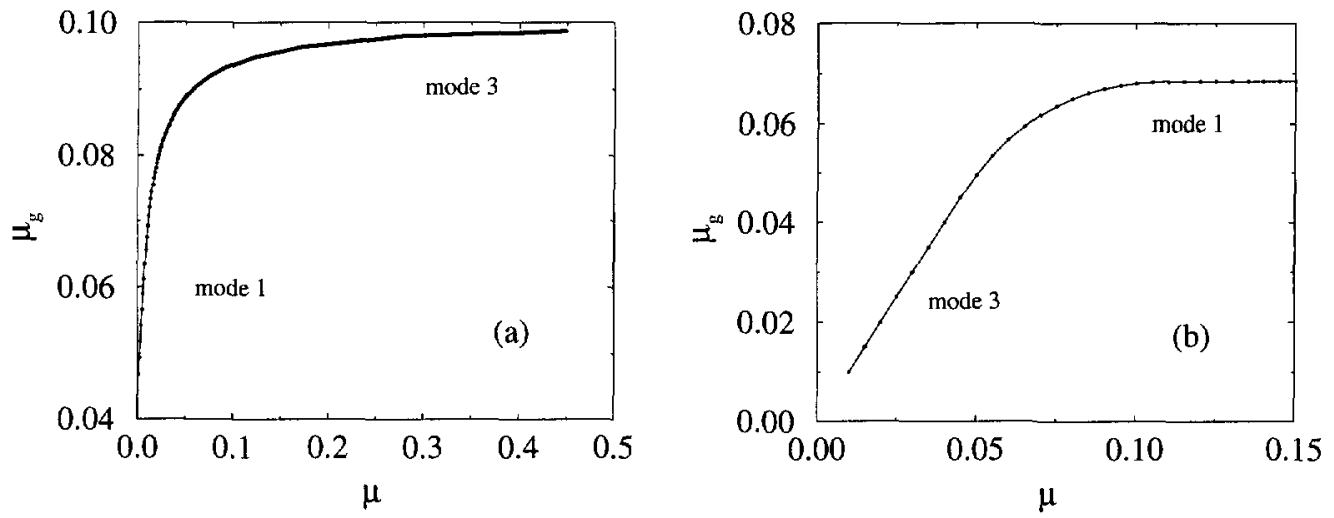

Fig. 12. - Steady-state global coefficient of friction $\mu_{\mathrm{g}}$ as a function of the particle-particle coefficient of friction $\mu$ for $\mu^{\prime}=0.1$ (a) and as a function of the particle-plane coefficient of friction $\mu^{\prime}$ for $\mu=0.01$ (b) in an array of 30 particles as simulated by the CD method. Parameters are $N_{L}=4, N_{0}=0.01$, and $\mu^{\prime \prime}=0.01$.

with $L$, the global coefficient of friction for the whole array increases and asymptotically tends to $\mu^{\prime}$. Again, we recall that at first order in $\mu$ the global coefficient of friction is independent of $L$ in all conditions.

6.3. Influence of Local Friction Coefficients. - When one of the coefficients of friction $\left(\mu, \mu^{\prime}\right.$ or $\left.\mu^{\prime \prime}\right)$ is changed, the system may still keep its configuration of modes (the number of particles in each mode). The effective coefficients of friction for each mode then change according to $(4,6)$ as a function of the coefficients of friction. In mode $1, \mu_{\mathrm{e}}$ increases with $\mu$, but is independent of $\mu^{\prime}$. In mode $2, \mu_{\mathrm{e}}$ increases both with $\mu$ and $\mu^{\prime}$. Finally in mode $3, \mu_{\mathrm{e}}$ does not depend on the coefficients of friction.

However, the values of friction coefficients also influence the configuration of modes along the array. Figure 12a shows $\mu_{\mathrm{g}}$ as a function of $\mu$ in the case where the three modes are present along the array and where all other parameters and the driving force are fixed. At low enough values of $\mu$, all particles are in mode 1 . $\mu_{\mathrm{g}}$ increases rapidly with $\mu$. The variation with $\mu$ slows down as the number of particles in modes 2 and 3 increases and tends asymptotically to $\mu^{\prime}$ for high enough values of $\mu$ for which all particles are in mode 3 .

Figure 12b shows $\mu_{\mathrm{g}}$ as a function $\mu^{\prime}$ for the same system. At low enough values of $\mu^{\prime}$ all particles are in mode $3 . \mu_{\mathrm{g}}$ is then equal to $\mu^{\prime}$ and increases with it. As $\mu^{\prime}$ increases, more and more particles appear in modes 2 and $1 . \mu_{\mathrm{g}}$ tends to its asymptotic value, as a consequence, when all particles are in mode 1 . Note that here $\mu_{\mathrm{g}}$ increases with $\mu^{\prime}$ although the number of particles in mode 1 increases.

Finally, the particle-block coefficient of friction $\mu^{\prime \prime}$ does not influence notably the modes and $\mu_{\mathrm{g}}$. Its major effect concerns the particle rotations in mode 3 which gives rise to spikes on the plots of the effective quantities as a function of $\dot{v}$.

\section{Conclusion}

The simple model of rigid particles we studied in this paper has two main properties: all contacts are mechanically persistent and particle rotations are frustrated as in a dense granular system. The frictional dynamics of such a system gives rise to nontrivial correlations. These correlations appear both in time, by the self-organization of dynamic variables to reach a steady state, and in space, by mesoscopic length scales involving collective rotation modes. 
This picture is somewhat modified as soon as molecular dynamics (MD) with a regularized version of Coulomb's law is used. The evolution of the system and of the friction forces is then dominated by elastic oscillations at short time scales and the steady state is sometimes reached only in a statistical sense at long time scale. Moreover, in the presence of particles for which all contacts are nonsliding the discrepancy between the two methods is likely to increase. But, as far as the steady state is concerned, we find globally very good agreement between the CD and MD results for this frustrated one-dimensional model.

The main difficulty with the MD method in the context of the present model is the fact that it does not provide a simple key to the description of the dynamics of the system. We showed that a nonsmooth friction law is inevitably bound to dynamics. Both the step-by-step loss of the memory of initial conditions and the collective modes result from this interplay between dynamics and friction. The concepts of nonsliding and persistent contacts in the dynamic sense are well-suited for a physical and transparent description of the behavior of the system. On the contrary, in the MD method only a careful checking (taking into account the numerical precision) of the effective values of velocities in each time step allows to establish the underlying phenomena.

With a friction law involving both a static coefficient of friction and a dynamic one, the steady state is dependent on initial conditions. However, we saw that it is possible to define global static and dynamic coefficients of friction with such a friction law if the system starts moving from static equilibrium. As a particular case, we showed that if the mechanical parameters are such that all particles are in the rolling mode, then the difference between these global coefficients of friction increases with system size.

To what extent are these results relevant to real granular systems? The phenomenology of a real disordered granular system is far more complex. The present study shows that part of this complexity should have its origin in the nonsmooth nature of interaction laws. The presence of nonsliding contacts naturally gives rise to long-range correlations and a collective organization of contact states. While the continuum classical description of granular systems does not take into account particle rotations, the rich physics of this simple $1 \mathrm{D}$ model suggests that they should play an important role in the dissipative evolution of a sheared granular material.

\section{Acknowledgments}

We gratefully acknowledge many fruitful discussions with L. Brendel and S. Roux. This work has been supported by the European program of Human Capital and Mobility, Contract No. CHRXCT 930354.

\section{Appendix A}

\section{Prescription of the Exact Coulomb's Law in Simulations}

The iteration process briefly described in Section 3.1 has to be simultaneously applied to all contacts. At the beginning, for a given configuration of particle velocities, the states are known only for contacts where $v^{\mathrm{t}} \neq 0$, which are all sliding (S). The activated contacts, i.e. contacts where $v^{\mathrm{t}}=0$, can be $\mathrm{S}$ or NS in the dynamic sense, i.e. $\dot{v}^{\mathrm{t}}=0$ or $\dot{v}^{\mathrm{t}} \neq 0$ respectively. We may start with a random distribution of states at such contacts and calculate dynamic variables. If they all satisfy the corresponding inequality at each contact, then the solution is acceptable. Otherwise, some states at active contacts are to be modified. Again, the dynamic variables are calculated with the new equations and checked with respect to the inequalities. This process is iterated until the solution is reached. So we see that through this iteration, which is only internal to a numerical code, we obtain both the values of the dynamic variables and the dynamic contact states. 
One peculiarity of the closed-contact $1 \mathrm{D}$ system is that, as mentioned in the introduction, for a wide range of mechanical parameters all particle-particle and particle-plane contacts stay closed. The closed-contact condition implies that the interstitial distance $d_{\imath \jmath}$ between two bodies $i$ and $j$, the normal relative velocity $v_{\imath \jmath}^{\mathrm{n}}$, and the relative normal acceleration $\dot{v}_{\imath \jmath}^{\mathrm{n}}$ all be zero. The last one is the condition of a persistent $(\mathrm{P})$ contact in the dynamic sense. It should be supplemented to the equations of dynamics. If it happens that at a given contact $\dot{v}_{\imath \jmath}^{\text {n }} \neq 0$, then that contact should open and it is a nonpersistent (NP) contact, even if $v_{\imath \jmath}^{\mathrm{n}}=0$.

A natural question raised by the method depicted above to prescribe Coulomb's law, is whether a solution exists. Let us consider a granular assembly of $p$ particles and $c$ contacts. We have, in two dimensions, $3 p$ equations of dynamics. On the other hand, there are $3 p$ accelerations and $2 c$ tangential and normal forces to be determined. For a given set of tangent contact states (S or NS) and normal contact states ( $\mathrm{P}$ or NP), there are $c$ equations given by the friction law and $c$ more equations given by the normal contact law. In the 1D array which we consider in this article, the normal contact law is the closed-contact condition, which should result in repulsive forces (otherwise the array is no more stable and the simulation has to be stopped). In this way, for a given set of contact states, the number of equations is equal to the number of unknowns, so that the system of equations has formally at least one solution.

The iteration process has, however, to converge to a mechanically acceptable solution, i.e. the contact states should be such that the inequalities corresponding to each alternative equation, listed in (1), be satisfied. From the description given above it is not obvious that such a mechanically acceptable solution should exist at all or that the solution should be unique. It can be shown that, for example, any loop of NS contacts implies a continuous set of solutions for contact forces. We will not discuss further such indeterminate states here; a more detailed discussion can be found elsewhere [12]. As far as the 1D model is concerned, we would like to mention that our simulations by the method described here, i.e. Contact Dynamics method [10], have always given a unique solution.

\section{Appendix B}

\section{Normal Forces in MD Simulations}

Contrary to the CD method, which models perfectly rigid particles that interact only if the interstitial distance $d_{\imath \jmath}$ is zero, in MD particles can overlap ("soft grains") and interact if $d_{23} \leq 0$. This overlap or interpenetration is a measure of elastic deformation at the contact region. A repulsive force with some kind of damping is implemented in the normal direction which keeps the overlap small and models the dissipation occurring in the impact of two particles. In long-lasting contacts, which we will discuss here, this damping mainly serves as a means of damping possible oscillations of the particles around their equilibrium distance, since in long-lasting contacts, the relative normal velocities of the particles should vanish. For the normal force, we use

$$
N=-k_{\mathrm{n}} d_{\imath \jmath}-\gamma_{\mathrm{n}} \dot{d}_{\imath \jmath}^{\mathrm{n}}, \quad d_{\imath \jmath} \leq 0,
$$

i.e. a linear spring with viscous damping, where the spring constant $k_{\mathrm{n}}$ determines the time step. $\gamma_{\mathbf{n}}$ mainly determines how fast oscillations resulting from the initial state are damped away and has no further significance here. We want to stress, however, that in simulations of granular flow, where particle contacts open and close, these parameters have a clear physical meaning and should be adjusted to realistic values [14]. For the simulations presented here, we chose $k_{\mathrm{n}}=2 \times 10^{5}$ and $\gamma_{\mathrm{n}}=200$ in terms of the basic units used in this paper. The limit of rigid particles can be approached by increasing $k_{\mathrm{n}}$. However, this increases the computation time, since the time step scales as $k_{\mathrm{n}}^{-1 / 2}$ In this limit, the CD method is naturally more convenient since it directly prescribes the condition of perfect rigidity $[10,11]$. 


\section{References}

[1] de Coulomb C., Mémoires de mathématiques et de physique (Imprimerie Royale, Paris) (1773) p. 343.

[2] Nedderman R.M., Statics and kinematics of granular materials (Cambridge University Press, 1992).

[3] B.J. Briscoe, in "Physics of Granular Media", D. Bideau and J. Dodds, Eds. (Nova Science, New York, 1991).

[4] Jaeger H.M. and Nagel S.R., Science 255 (1992) 1523.

[5] Dantu P., in Proc. 4th Int. Conf. Soil Mechanics and Foundations Engineering, London (1957).

[6] Liu Y, Nagel S.R., Schecter D.A., Coppersmith S.N., Majumdar S., Narayan O. and Witten T.A., Science 269 (1995) 513.

[7] Radjai F., Roux S., Jean M. and Moreau J.J., Phys. Rev. Lett. 77 (1996) 274.

[8] Radjai F. and Roux S., Phys. Rev. E 51 (1995) 6177.

[9] Heslot F., Baumberger T., Perrin B., Caroli B. and Caroli C., Phys. Rev. E 49 (1994) 4973-4988.

[10] Moreau J.J., Eur. J. Mech. A/Solids 13 (n 4-suppl.) (1994) 93-114.

[11] Jean M., in "Mechanics of Geometrical Interfaces", A.P.S. Selvadurai and M.J. Boulon, Eds. (Elsevier Science B.V., 1995) pp. 463-486.

[12] Radjai F., Brendel L. and Roux S., Phys. Rev. E 54 (1996) 861.

[13] Wolf D.E., in "Computational Physics: Selected Methods - Simple Exercises - Serious Applications" K.H. Hoffmann, M. Schreiber, Eds. (Springer, Heidelberg, 1996).

[14] Schäfer J., Dippel S. and Wolf D.E., J. Phys. I France 6 (1996) 5.

[15] Thompson P.A. and Grest G.S., Phys. Rev. Lett. 67 (1991) 1751.

[16] Ristow G.H., J. Phys. I France 2 (1992) 649.

[17] Pöschel T., J. Phys. II France 3 (1993) 27.

[18] Melin S., Phys. Rev. E 49 (1994) 2353.

[19] Pöschel T., J. Phys. I France 4 (1994) 499.

[20] Pöschel T., Herrmann H.J., Europhys. Lett. 29 (1995) 123.

[21] Dippel S., Batrouni G.G. and Wolf D.E., to appear in Phys. Rev. E (1996).

[22] A probably more dramatic error results from the sign of the friction force. At a NS contact, the sign of the friction force can be positive or negative, depending on the dynamics. The $\mathrm{RC} 1$ law always gives a friction force directed opposite to the relative velocity, even when the relative velocity is not physically significant.

[23] This means that no contact will become activated any more, so that the contact states cannot change.

[24] Radjai F., Evesque P., Bideau D. and Roux S., Phys. Rev. E 52 (1995) 5555.

[25] This behavior can be understood in terms of rotation modes. At low values of $N_{L}$ all particles of the array are in mode 1 . Since the friction forces at particle-plane contacts are only partially mobilized, the global friction force is small. We should emphasize here the point that, although all particle-plane contacts are nondissipative in this mode, the global array-plane friction does measure the dissipation rate in the system. In fact, the mobilization of friction forces at particle-plane contacts is directly related to dissipation at particle-particle contacts. The normal particle-particle forces increase with the driving force, so that interparticle dissipation, friction forces at particle-plane contacts and, as a consequence, $\mu_{\mathrm{g}}$ increase. 